\title{
Crosstalk of Autophagy and the Secretory Pathway and its role in diseases
}

Muhammad Zahoor ${ }^{1}$, Hesso Farhan ${ }^{1 \#}$

1 Institute of Basic Medical Sciences, University of Oslo, Norway

\# Corresponding author: hesso.farhan@medisin.uio.no

\begin{abstract}
The secretory and autophagic pathways are two fundamental, evolutionary highly conserved endomembrane processes. Typically, secretion is associated with biosynthesis and delivery of proteins. In contrast, autophagy is usually considered as a degradative pathway. Thus, an analogy to metabolic pathways is evident. Anabolic (biosynthetic) and catabolic (degradative) pathways are usually intimately linked and intertwined, and likewise, the secretory and autophagy pathways are intertwined. Investigation of this link is an emerging area of research and we will provide an overview of some of the major advances that have been made to contribute to out understanding of how secretion regulates autophagy and vice versa. Finally, we will highlight evidence that supports a potential involvement of the autophagy-secretion crosstalk in human diseases.
\end{abstract}




\section{The secretory pathway}

The cytosol of eukaryotic cells is subdivided into many membrane-bound compartments, which allow these cells to spatially separate diverse cellular processes such as translation, transcription, or ATP synthesis. In addition, it allows cells to increase their membrane area without substantially increasing cell size. However, compartmentalization created the need to develop processes that allows organelles to exchange lipids and proteins, and to allow organelles to deliver their content to the extracellular milieu. This process of delivering and exchanging membranes and proteins is called secretion, and is one of the many functions of the secretory pathway.

The secretory pathway comprises an array of membrane-bound organelles that communicate via vesicular or tubular carriers (Fig. 1A). Groundbreaking work in the past decades has led to a profound understanding of the machinery that orchestrates protein trafficking between organelles. The original yeast genetics screens by the Schekman group identified (sec) mutants that exhibit defects in secretion (Novick and Schekman 1979; Novick et al. 1980). This screen provided us with a collection of genes that regulate various steps of protein biogenesis and trafficking. Secretory proteins are delivered to or integrated into the endoplasmic reticulum (ER) in a manner dependent on the signal recognition particle. The ER is a network composed of tubules and cisternae that extend from the nuclear envelop throughout the entire cytoplasm. The ER hosts a wide range of chaperones that assist in folding of newly synthesized proteins. These chaperones also act as quality control (QC) machinery that prevents the release of misfolded or immature proteins from the ER. The journey of secretory proteins begins after their release from the QC machinery, when they are packaged into COPII vesicles, which form at specialized ribosome-free regions of the rough ER called the ER exit sites (ERES; or alternatively referred to as transitional ER). The number of ERES per cell varies depending on the organism and the cell type. Mammalian cells typically have few hundred ERES that are distributed throughout the cell. They were shown to form de novo and to undergo fusion and fission and to respond to differences in cargo load (Stephens, 2003; Farhan et al, 2008). ERES represent as clusters of tubules and vesicles on the ER and are currently viewed as the sole sites for the biogenesis of COPII vesicles. As will be discussed later they are gaining increasing attention as important sites for autophagosome biogenesis. 
The core machinery required for a COPII coat is composed of five subunits: Sar1, Sec23, Sec24, Sec13 and Sec31 (Fig. 1A). The initial step of COPII coat assembly is the recruitment of the small GTPase Sar1 by Sec12, its guanine nucleotide exchange factor (GEF) (Barlowe \& Schekman, 1993). Sec12 is a type-II transmembrane protein, which was recently shown to concentrate at ERES in a manner dependent on cTAGE5 and TANGO1 (Raote et al, 2017). After activation of Sar1 by Sec12, the small GTPase associates with the ER membrane and recruits the hetero-dimeric Sec23/Sec24 complex. Sec23 (of which there are two isoforms in mammals) acts as a GTPase activating protein (GAP) for Sar1. Sec24 (of which there are 4 isoforms in mammals) acts as an adaptor for secretory cargo. Several binding sites on Sec24 in yeast and mammals were described to mediate the selective export of cargo from the ER (Miller et al, 2002; Miller et al, 2003; Farhan et al, 2004; Mancias \& Goldberg, 2008). The Sar1-Sec23-Sec24 complex is often referred to as the pre-budding complex. Subsequently, a Sec13-Sec31 hetero-tetramer is recruited which forms the outer layer of the COPII coat, leading ultimately to budding of a COPII coated vesicle (Fig. 1A).

In yeast, COPII vesicles ferry cargo directly to the Golgi. However, in mammalian cells, ER-derived secretory cargo passes first through the ER-Golgi intermediate compartment (ERGIC), which represents as a cluster of vesicles and tubules (Appenzeller-Herzog \& Hauri, 2006). The ERGIC has been long debated for whether it represents a stable compartment, or whether it is merely a collection of carriers on their way to the Golgi. However, evidence from live cell imaging experiments (Ben-Tekaya et al, 2005; Jarvela \& Linstedt, 2012) as well as recent reports on the role of the ERGIC in autophagy (see below) suggest that the ERGIC is a genuine compartment that is located along the bidirectional ER-Golgi trafficking route. It is still enigmatic how secretory cargo leaves the ERGIC towards the Golgi.

The Golgi is the last station of what we know as the early secretory pathway. In most cell types the Golgi represents as a stack of flattened cisternae that are divided into three subcompartments, namely the cis-, medial-and trans-Golgi. In plants, worms, flies, or Pichia, several such Golgi stacks exits, typically in close vicinity of the ERES forming a secretory unit. In mammalian cells, several Golgi stacks connect via lateral anastomoses to form the Golgi ribbon. In light microscopy, the Golgi ribbon appears as a single copy organelle located in the center of the cells closely associated with the centrosome. The Golgi is a major hub for post-translational protein modification, for protein and lipid sorting and for cellular signaling. Moreover, the Golgi plays an important role in cell polarity and cell migration. 


\section{Autophagy}

Autophagy is a term referring to a self-eating process, introduced for the first time by de Duve at Ciba foundation symposium in 1963. Although the term "autophagie" was coined a century earlier by M. Anselmier to describe the response of animals to starvation (Ktistakis, 2017), it was de Duve who was the first to use it to describe a cellular process whereby a membrane-bound structure (the autophagic vacuole) engulfs recognizable cytoplasmic components (De Duve \& Wattiaux, 1966). The term autophagosome was later introduced to describe vesicular elements associated with what was then called the autophagic vacuole. Groundbreaking yeast genetic studies in the 1990s identified the Atg genes as the machinery that regulates biogenesis and trafficking of autophagosomes (Takeshige et al, 1992). Orthologs of ATG genes in different species were subsequently identified leading to fairly solid understanding of the fundamental steps of autophagy, which will be introduced very briefly below.

Autophagy may be classified into three main classes: macroautophagy, microautophagy, and chaperone-mediated autophagy (CMA). CMA describes the translocation of cytosolic proteins across the lysosomal membrane and microautophagy involves the uptake of cytosolic proteins into lysosomes by invagination. The bestcharacterized type of autophagy that will be dealt with in this review is macroautophagy (hereafter referred to as autophagy), of which two basic subtypes exist: non-selective (bulk) and selective autophagy. Both subtypes share a common hierarchically organized machinery (Fig. 1B). Non-selective autophagy is a process where cells engulf a portion of their cytoplasmic content in response to deprivation of nutrients (Mortimore \& Schworer, 1977; Takeshige et al, 1992). Thereby cells are able to recycle building blocks (e.g. amino acids) and compensate for the lack of nutrients. Being a bulk response to starvation, autophagy was considered non-selective toward its substrates. However, it is now clear that autophagy also occurs under homeostatic conditions, in the absence of any nutrient deprivation. This response is specific to degradation of selected cellular components and therefore called selective autophagy (Fig. 1C). Depending on the type of cargo, selective autophagy mediates the degradation of protein aggregates (Aggrephagy), damaged organelles such as peroxisomes 
(Pexophagy), the ER (ER-phagy) or mitochondria (Mitophagy) (Farré \& Subramani, 2016; Svenning \& Johansen, 2013).

The first step in building an autophagosome is the formation of double-membrane structure called the phagophore or isolation membrane, which will grow and engulf (selectively or unselectively) the autophagic cargo. In yeast, this tales place at the phagophore assembly site (PAS). In mammals the closest analogous structure is the omegasome (Axe et al, 2008), a PI3P positive structure that stains positive for a protein called DFCP1. Autophagosome biogenesis is orchestrated by a conserved machinery that is subdivided into different, mostly cytosolic, complexes that are recruited in a hierarchical manner during the biogenesis of autophagosomes (Fig. 1B). This machinery interacts with membranes, phosphorylates and shapes them to generate an autophagosome (Carlsson \& Simonsen, 2015). There are two initiation complexes that are of central importance: the ULK1 (unc-51-like autophagy activating kinase 1) complex (Atg1 in yeast) and the class III PI 3-kinase complex I (PI3KC3-C1). Mammalian ULK1 belongs to a family of four Ser/Thr kinases (ULK1-4) and forms a complex together with the non-catalytic subunits ATG13, ATG101, and FIP200. In yeast Atg1 forms a complex with Atg13, Atg17 and the yeast-specific subunits Atg29 and Atg31. The activation of the ULK1/Atg1 complex is still a subject of intense investigation, but there is consensus that inhibition of mTORC1 is a strong stimulus.

The class III phosphatidylinositol 3 kinase complex is recruited immediately downstream of ULK1/Atg1. The complex is composed of the PI3-kinase Vps34/PIK3C3, Atg14/ATG14L, Atg6/Beclin-1, and Vps15/PI3R4. Formation of PI3P on the ER is required for recruitment of WIPI2 (Atg18 together with Atg2 in yeast). This paves the way for conjugation of ATG8 (six members in mammals and one in yeast) to phosphatidylethanolamine (PE) in the isolation membrane. This is achieved via the ATG12 conjugation complex composed of ATG12, ATG5 and ATG16L1.

Non-selective autophagy may be divided into initiation, elongation/expansion of the isolation membrane followed by completion and subsequent fusion of the autophagosome with the lysosome. Selective autophagy requires an additional step where an autophagy receptor is recruited that mediates the sorting of the autophagic cargo into the autophagosome. The first selective autophagy receptor was the mammalian protein p62 (SQSTM1) (Bjørkøy et al, 2005; Pankiv et al, 2007) and was shown to be responsible for the degradation of ubiquitylated proteins. Later, p62 was also shown to be involved in the autophagic clearance 
of Salmoenlla (Zheng et al, 2009). This autophagy receptor contains an ubiquitin-binding domain to recognize ubiquitylated cargo destined for degradation. In addition, it contains a LC3-interaction region (LIR) that mediates the interaction with the nascent autophagosome. A wide range of LIR-containing autophagy receptors was identified and shown to mediate different types of selective autophagy (for a detailed list see Svenning \& Johansen, 2013). Thus, the ability to bind to LC3 appears to be crucial for linking cargo and the autophagosome. While there is only one Atg8 isoform in yeast, mammals have six Atg8 family members known as the light chain 3 (LC3) or $\gamma$-aminobutyric acid (GABA)-receptor-associated protein (GABARAP) proteins (Slobodkin and Elazar, 2013; Weidberg et al, 2010). It remains to be fully understood how these different LC3 variants contribute to the specificity of selective autophagy. A role for the autophagic cargo itself in regulating (or even inducing) autophagy has recently been discussed (Zaffagnini \& Martens, 2016). Finally, selective autophagy was also shown to be regulated by kinase signaling as in the case of the autophagy receptor optineurin. As other receptors, optineurin contains a LC-3 interacting domain as well as an ubiquitin-binding region. Upon infection with Salmonella, inflammatory signaling phosphorylates optineurin, which increases its ability to bind ubiquitiylated cargo (Wild et al, 2011).

\section{The link between autophagy and the secretory pathway}

In analogy to metabolic pathways, the secretory pathways may be compared with of as a biosynthetic (i.e. anabolic) process, while autophagy could be considered a degradative (i.e. catabolic) process. Anabolic and catabolic pathways are usually intimately linked and intertwined, and likewise, there is mounting evidence that the secretory and autophagy pathways are closely associated. Two features are recurrent when considering this link: firstly, membranes of the secretory pathway serve as sources and platforms for autophagosome biogenesis. Secondly, there are several proteins that are shared between these two pathways and we have coined the term "multi-tasking proteins" to describe this phenomenon (Farhan et al, 2017). Below, we will review the role of the secretory pathway as a source or platform for autophagosome biogenesis with emphasis on the ER, ERES, ERGIC and the Golgi. We will also highlight for each of these compartments proteins that multitask between both endomembrane pathways. 


\subsection{The ER as a source for autophagosomes}

The secretory pathway represent the largest source of membranes within the cell. The quest for the source of membranes for autophagosome biogenesis kept the scientific community busy ever since. The ER is the largest organelle in cells and therefore, it is not surprising that this organelles was the first that was considered a membrane source of autophagosomes. Before, the ER was clearly implicated in the biogenesis of autophagosomes, two models were discussed: the assembly model suggested that autophagosomes assemble de novo from localized lipid synthesis or transport. On the other hand, the maturation model suggests that autophagosomal membranes originate from the ER (or another organelles) (Juhasz \& Neufeld, 2006). Nowadays, there is compelling evidence that the ER is a major source of autophagosomes. Using imaging experiments, several autophagic proteins were found to localize to the ER, to be active there and to undergo cycles of binding and unbinding, implying active recruitment. Isolation membranes were found to emerge from an omegasome (named because of their omega-shape), and to recruit DFCP1, a protein with a FYVE domain that allows it to bind PI3P enriched membrane regions (Axe et al, 2008). Besides live imaging microscopy of fluorescent protein, electron tomography provided further strong evidence for the involvement of the ER in autophagosome formation (Hayashi-Nishino et al, 2009; YläAnttila $\mathrm{P}$ et al, 2009). A subdomain of the ER was shown to form a curved structure that encircles the isolation membrane between two ER cisternae. Notably, this structure was continuous with the ER network, which led the authors to suggest that this ER subdomain acts as a 'cradle' for the biogenesis of the autophagosome. Later, this structure was shown to be positive for DFCP1 (Hamasaki et al, 2013). This tempts to speculate that this "cradle" is identical to the omegasome, but this has to be formally demonstrated. Another protein enriched at this structure is ATG14L (also called Barkor), which is part of the PI3-kinase complex and represents the targeting factor for PI3KC3 to the autophagosome membrane (Matsunaga et al, 2010). ATG14L uses a hydrophobic amphipathic helix to bind to autophagosomes. Importantly, this domain was proposed to induce curvature (Fan et al, 2011), thereby linking the formation of a PI3P-rich membrane patch to induction of curvature that might be important for formation of the isolation membrane or the omegasome. The ULK1 kinase was also shown to associate with the growing omegasome and to dissociate from it after it reached a certain size (Karanasios et al, 2013). These cycles of binding and dissociation imply a regulated mode of recruitment of ULK1 to this ER subdomain. ULK1 was long thought to be selective for autophagy. Recently, the Kundu group showed that 
ULK1/2 are capable of phosphorylating Sec16A, a scaffold protein that facilitates the biogenesis and maintenance of ERES (Sprangers and Rabouille, 2015; Joo et al, 2016). This phosphorylation was shown to be required for ER export of a specific set of cargo proteins. Sec16A phosphorylation by ULK1/2 occurred under condition where autophagy was not induced, indicating that this represents a novel function of ULK1 unrelated to autophagy. Future studies are needed to determine why and how the ULK1-Sec16A signaling axis controls exit of a selective set of cargos and whether it plays any role in autophagy. ULK1 was more recently found to phosphorylate the COPII component Sec23A (Gan et al, 2017). Contrary to its effect on Sec16A, ULK1 phosphorylated Sec23A mainly under conditions of active autophagy and this phosphorylation was linked to a morphologic change of ERES under amino acid starvation (i.e. activation of macroautophagy). ULK1 phosphorylates Sec23A on two residues, which regulates its interaction with Sec31A. The conclusion from these two papers might be that a switch from anabolic to catabolic conditions redirects ULK1 from a pro-secretion kinase (via Sec16A phosphorylation) towards an autophagy regulating kinase that shuts down secretion (Joo et al, 2016; Gan et al, 2017). Whether this interpretation is true and how this switch is regulated remains a topic for future investigations on this "multitasking" role of ULK1/2. In addition, it will be important to elucidate whether there are two distinct pools of ULK1/2, or whether a common pool of these kinases regulates both autophagy and/or secretion.

The ER is known to form contact sites with multiple other organelles and membranes. These contact sites were also shown to act as potential nucleation sites for autophagosomes. ER-mitochondria contact sites (ERMCS) are physical contacts between these two endomembranes, which are important for various processes such as mitochondrial fission or lipid transfer (Rowland \& Voeltz, 2012). Using a combination of live fluorescence imaging and electron microscopy, ATG14L was found to localize to ERMCS upon the induction of autophagy in a manner dependent on the ER-resident SNARE protein syntaxin 17 (Hamasaki et al, 2013). More recently, ER-plasma membrane contact sites were shown to act as platforms for the biogenesis of autophagosomes. Markers of autophagy are recruited to sites enriched in the tethering extended synaptotagmins (E-Syts), which are located on ER-PM contact sites (Nascimbeni et al, 2017). Silencing of E-Syt expression negatively affected autophagosome biogenesis. This supports the notion that autophagosomes are not only generated on the ER itself, but that contact sites of this organelles with other membranes are of relevance for autophagosome biogenesis. 


\subsection{ERES and COPII components in autophagosome biogenesis}

Exit from the ER occurs at ERES, which exhibit constant formation of transport carriers. The observation that functional ERES are important for autophagosome formation led to the identification of a number of proteins that play a dual role in autophagy and ER exit. For instance, the small GTPases Rab1 (Ypt1 in yeast), which was shown to be important for both processes, exerts its dual role at or in close vicinity to ERES (Zoppino et al , 2010). Saccharomyces cerevisiae strains with defects in genes important for COPII vesicle formation exhibit impairment in the ability to generate autophagosomes (Ishihara et al., 2001; Reggiori et al., 2004). The same appears to be true in mammals, where alteration of the function of the small GTPase Sar1 results in strong defects in autophagy (Zoppino et al , 2010). This defect appears to be at very early stages of autophagy, because alterations of COPII function decreases the number of puncta positive for DFCP1, indicating a defect in omegasome formation. Recently, two models were proposed to explain the role of ERES in autophagy (Sanchez-Wandelmer et al, 2015). According to model-I, ERES act as donors of membranes via vesicular traffic (Fig. 2). In model II ERES serve as a platform or scaffolds for autophagosome biogenesis (Fig. 2). Strong support for model-I comes from work in yeast on the roles of TRAPP complexes and Ypt1 and we will therefore briefly introduce these. The small GTPase Ypt1 (Rab1A in mammals) is regulated by three different multiprotein complexes that act as a GEF. These are referred to as the transport protein particle I (TRAPPI), TRAPPII and TRAPPIII. TRAPPI regulates ER-to-Golgi transport by mediating tethering of COPII vesicles (Cai et al, 2007), TRAPPII regulates intra-Golgi transport (Yamasaki et al, 2009) and TRAPPIII appears to be the autophagy specific GEF for Ypt1 (Lynch-Day et al, 2010). Depending on which TRAPP complex it associates with, the action of Ypt1 can be targeted towards autophagy or secretion. The TRAPP complex is composed of shared "core" subunits as well as subunits specific to the individual complexes. Thus, it is conceivable that the specificity of a TRAPP complex for autophagy or secretion may change by simply exchanging a single subunit. The TRAPPIII specific subunit Trs 85 guides the complex to the phagophore assembly site to locally activate Ypt1 (Lynch-Day et al, 2010), recruit Atg1 (ULK1 in mammals) and to initiate the formation of the phagophore (Wang et al, 2013).

Taking together the role of TRAPP complexes in COPII vesicle tethering and in autophagy, we might speculate that ERES act as donors of membranes (COPII vesicles) to 
which TRAPPIII is recruited, which in turn will activate Ypt1 and Atg1. A recent paper by the Ferro-Novick group further supports this model, by providing a mechanism how COPII vesicles are diverted towards autophagy. During autophagy, Sec24 becomes phosphorylated, which facilitates its interaction with Atg9 (Fig. 2), a transmembrane protein required for the initiation of autophagy (Davis et al, 2016). These ATG9 containing membranes may either be already formed autophagosomes, or are precursors for autophagosomes. Notably, Atg9positive vesicles were shown to be able to recruit TRAPPIII (Kakuta et al., 2012). Finally, ER-derived membranes positive for the yeast SNARE protein Ufe1 were shown to be delivered to Atg9-positive structures (Lemus et al, 2016).

The membrane donor model (referred to as model-I above) appears to be strongly supported by data obtained from work in yeast. However, the situation is less clear in mammals. While the autophagy-specific role of TRAPPIII in yeast was well established, its role in mammals remained poorly understood, mainly because the composition of mammalian and yeast TRAPPIII are not identical, because the yeast Trs85 subunit is absent in mammals. TRAPPC12, which has no yeast ortholog, was proposed as a TRAPPIII specific subunit in addition to TRAPPC8 (Behrends et a, 2010). Recently, TRAPPIII was shown to exert clear functions on the secretory pathway. Depletion of TRAPPC12 resulted in a reduction in the number of ERES per cell, dispersion of the Golgi and the ERGIC and an inhibition of ER-toGolgi transport (Zhao et al, 2017). Depletion of TRAPPC12 was also shown to negatively affect autophagy (Behrends et al, 2010). Thus, in mammals TRAPPIII appears to have functions on both the autophagic and the secretory pathway. Given the importance of ERES for autophagy (Zoppino et al , 2010), the question arises of whether the initially observed effects of TRAPPIII on autophagy are indirect consequences of its effects on ERES. An interesting observation was that the effect of TRAPPC12 depletion on the ERGIC could be partially rescued by an inhibition of autophagy, indicating that TRAPPC12 depletion primarily affects ERES function, while autophagy still continues to consume the ERGIC. Subsequent defects in autophagy (observed at later stages) are then a consequence of alterations of ERES function. Clearly more work is needed to clarify the precise role of TRAPPIII (and other TRAPP complexes) in autophagy and secretion. In addition, a phosphorylation-dependent re-routing of COPII vesicles for autophagy has yet to be demonstrated for mammalian Sec24 in analogy to yeast (Davis et al, 2016). Yeast Sec24 was phosphorylated on 3 threonine residues (T324/T325/T328), but these are moderately conserved in mammalian Sec24A (2 out of 3 residues), but not in the other Sec24 isoforms. 
Moreover, ERES in mammalian cells are morphologically very different from yeast. In mammalian cells, ERES are ribosome-free regions of the rough ER that are about $600 \mathrm{~nm}$ in diameter (Zeuschner et al, 2006), while in yeast ERES are defined as stochastic sites where COPII vesicles are formed. Thus, we might assume that ERES in mammals may represent platforms for the assembly of the autophagy machinery, which is in line with the aforementioned model-II (Sanchez-Wandelmer et al, 2015). The two models (ERES are either membrane donors or assembly platforms) might be considered alternative, mutually exclusive explanations for the role of ERES in autophagy. However, we favor the view that both pathways coexist in cells. Using a proteomic approach to map the interactome of core autophagy and COPII components, several interactions between these two machineries were identified indicating that physical and functional links between autophagy and ER export may exist (Graef et al, 2013). Therefore, we might speculate that upon induction of autophagy, the COPII components (and associated proteins such as Sec16) serve as recruiters for the autophagy machinery to the ER membrane. This is supported by the notion that COPII components are also capable of interacting with TRAPP complexes. Another example of a multitasking regulator is tectonin $\beta$-propeller-containing protein 2 (TECPR2), which regulates autophagy by binding to LC3 (Behrends et al., 2010; Oz-Levi et al., 2012). Recently, TECPR2 was shown to interact with Sec24D and to regulate ERES number as well as ER-toGolgi trafficking (Stadel et al, 2015). This finding could also be interpreted such that TECPR2 forms a platform at the ER, switching between being an ER export or autophagy regulator, but the mechanism how this switch is regulated remains to be determined in the future. Finally, an elegant study used live cell imaging, super-resolution microscopy and correlative light electron microscopy to investigate the earliest detectable autophagy-related structure, a punctum positive for ULK1 and ATG13. The authors showed that this ATG13 punctum forms on ER regions that later become positive for ATG9 vesicles (Fig. 2) and that this structure is dependent on functional ER export (Karanasios et al, 2016). However, another observation was that only $30 \%$ of these autophagy nucleation sites emerged in association with the ERES marker Sec16A, suggesting that ERES only partially account as platform for autophagosome nucleation (Karanasios et al, 2016). Thus, it is very likely that both models are true, namely, that ERES act as platforms for autophagosome assembly as well as donors of membranes for autophagy.

The coordination of secretion and autophagy is likely to occur at the level of signaling. ERES components were shown to be phosphorylated by several kinases that regulate the 
number and function of ERES. The atypical mitogen activated protein kinase MAPK15 was shown to negatively regulate secretion under starvation, in a manner dependent on signaling to Sec16 (Zacharogianni et al, 2011). MAPK15 was also shown to induce autophagy through direct interaction with LC3 (Colecchia et al, 2012). Thus, MAPK15 plays a role in turning secretion down, and inducing autophagy under catabolic conditions.

\subsection{The role of the ERGIC in autophagosome biogenesis}

As mentioned earlier, the ERGIC is a compartment that was found so far only in mammalian cells and is located on the crossroad of bidirectional ER-Golgi traffic. The identity of the ERGIC as a genuine compartment was controversially discussed, which partly stem from the fact that it was difficult to assign a function to it besides being a sorting station. In recent years, the ERGIC has become increasingly appreciated as a source for autophagosomes. Using a cell-free assay on fractionated membranes to determine which organelle support lipidation of LC3, the ERGIC was found to represent a major source of autophagosomes in vitro (Ge et al, 2013). Conditions that disrupted ERGIC formation, also negatively affected autophagy. However, it has to be stressed that these were all conditions that negatively affect COPII/ERES function. Thus, the observation that ERES might act as donors of membranes for autophagosomes might simply be due to the fact the ERES generate the ERGIC, which in turn donates membranes for autophagosomes. Such an interpretation is further corroborated by more recent findings by the same group. Using super-resolution microscopy, the authors find that ERES and the ERGIC undergo structural rearrangements that lead to a relocation of Sec12 (the GEF for Sar1) to the ERGIC (Ge et al, 2017). The authors suggest that this might lead to biogenesis of COPII vesicles from the ERGIC under conditions of active autophagy. While this model appears thought provoking, an alternative possibility might be that the ERGIC collapses back to ERES under autophagy conditions, making this new ERES/ERGIC hybrid a source for autophagosomes. In fact, it was recently shown that ERGIC membranes are recruited to ERES when these are challenges with collagen (Santos et al, 2015). This shows the principle ability for such a coalescence between ERES and ERGIC, and opens the possibility that this might also happen under conditions of active autophagy. 


\subsection{The role of the Golgi and Golgi-derived vesicles in autophagosome biogenesis}

Proteins arriving to the Golgi from the ER/ERGIC are either re-routed back to the ER in COPI vesicles, or may traffic to post-Golgi compartments such the endolysosomal system, the plasma membrane or the extracellular space. A role for components of the COPI coat has been proposed in the regulation of autophagosome biogenesis (Karanasios et al, 2016). The quest for this has been motivated based on the observation that blocking ER export with FLI06 (Krämer et al, 2013) only partially attenuated the formation of ATG13 puncta (i.e. autophagosome nucleation sites) (Karanasios et al, 2016). The effect was much more pronounced when combined with an inhibition of COPI function through treatment with brefeldin A or the knockdown of coatomer components. Alteration in the function of COPI alone resulted in a slight reduction in the number of starvation-induced autophagosomes, but the amount of lipidated LC3 increased, which suggests that COPI depletion affects the flux of autophagosomes (Karanasios et al, 2016). The authors went to analyze this phenomenon in greater detail and found that COPI plays a role in the elongation of autophagosomes by promoting the assembly or recruitment of the lipidation machinery for LC3. Strikingly, WIPI2 was misdirected to ring-like structures that did not associate with autophagosomes in starved cells. A general problem in all experiments that rely on alteration of COPI is that many of its effects are potentially indirect consequences of alterations of ERES. For instance, treatment with brefeldin A increases cargo load at ERES and increases their size (Farhan et al, 2008) and thereby might alter the ability to generate autophagosomes because the ERES become "busy" dealing with the challenge of increased cargo. Another consequence of the loss of COPI function is the alteration of early endosome function, which has been causally linked to an inhibition of autophagy (Razi et al, 2009). Thus, COPI trafficking appears to regulate autophagy through multiple mechanisms. However, does autophagy regulate COPI trafficking? So far, this is only a theoretical possibility, but there is some evidence in the literature to suggest that this might be the case. The PI3P-binding protein UVRAG localizes to the ER of unstressed cells, where it assembles into a RINT-1-containing tethering complex that regulates the arrival of COPI vesicles to the ER (He et al., 2013). Activation of autophagy results in dissociation of UVRAG from the RINT-1 complex, and subsequent mobilization of ATG9 from the Golgi (He et al., 2013). Thus, the function of the COPI arrival structure to the ER might be compromised under autophagic conditions, but this has to be studied in greater detail. Upon infection with bacterial pathogens GABARAPs, in particular GATE-16, were 
shown to interact with and activate Arf1, the GTPase that regulates COPI vesicle biogenesis (Sasai et al, 2017).

ATG9 is the only multispanning transmembrane protein among the autophagy core machinery proteins and as such is thought to be involved in arriving in vesicles to preautophagosome structures. ATG9-positive vesicles are derived from trans-Golgi network (TGN), and are known to be necessary for autophagy. In yeast, Atg9 becomes part of the autophagosome, but is recycled before the autophagosome fuses with the vacuole (Yamamoto et al., 2012). Yeast Atg9 is also directly phosphorylated by Atg1 (ULK1 in mammals), which has been shown to be necessary for biogenesis of autophagosomes (Papinski et al, 2014). In mammals, ATG9 does not seem to become part of the autophagosome itself (Orsi et al, 2012). Rather, ATG9 is proposed to deliver membranes from the Golgi and the early endosome towards sites of autophagosome biogenesis. Of note, these ATG9 vesicles appear to be targeted to ER, which itself is proposed to act as a membrane donor for autophagy. Future work needs to clarify the functional importance of these Golgi-derived membranes at the ER, which might be in supplying a different type of (phopho)lipids. Recently, these ATG9containing vesicles have been immunoisolated and found to be smaller than other vesicles involved in ER-Golgi trafficking (e.g. ATG9 vesicles are on average $42 \mathrm{~nm}$ in diameter while COPII vesicles are 60-80 nm). Proteomic analysis of these vesicles showed that they contain Rab1B, which is absent from the TGN and endosomal compartment, and indicates that it is acquired along the trafficking route of ATG9 vesicles towards the site of autophagy induction (Kakuta et al, 2017). This is supported by the finding that Rab1B is required for phagophore formation (Kakuta et al, 2017). Our understanding of the precise role of ATG9 in autophagy is further complicated by the finding that ATG9 traffics from the plasma membrane to the recycling endosome (Puri et al, 2013). There, ATG9 containing membranes fuse with the ATG16L1 compartment and thereby ATG9 helps ATG16L1 exit the recycling endosome and thus destines these membranes for the biogenesis of autophagosomes (Puri et al, 2013).

A connection between the Golgi and autophagy was recently postulated in the context of hepatitis $\mathrm{C}$ virus (HCV) infection. HCV entry into cells activates the Golgi localized immunity-related GTPase M (IRGM), which results in Golgi fragmentation (Hansen et al, 2017). IRGM also activates ULK1 and thereby induces autophagy, which provides the first evidence for a Golgi-based signaling regulator that activates autophagy (Hansen et al, 2017). Structural integrity of the Golgi might be directly linked to autophagy based on the observation that the Golgi matric protein GM130 interacts with the Atg8 homolog 
GABARAP, which renders it unavailable for autophagy. Upon starvation, GM130 binds to a protein called WAC, which sets GABARAP free and thus available to reach the phagophore.

The localization of mammalian ATG9 to the Golgi was recently shown to be regulated by kinase signaling. The N-terminus of ATG9 was shown to interact with the adaptor protein complexes AP1/2 (Zhou et al, 2017). In the absence of this interaction, ATG9 is misrouted to the plasma membrane. Notably, the AP1/2-ATG9 interaction is regulated by phosphorylation by the SRC tyrosine kinase and accordingly, EGFR-mediated activation of SRC promoted localization of ATG9 to the TGN (Zhou et al, 2017). However, this phosphorylation alone is not sufficient to regulate autophagy, but requires an additional phosphorylation of ATG9 by ULK1 (Young et al, 2006; Zhou et al, 2017).

\section{Secretory autophagy}

The links between secretion and autophagy were so far discussed exclusively for the conventional secretory pathway, which deals with proteins that harbor a signal peptide and are therefore capable of entering the ER. While this accounts for roughly a third of the cellular proteome, there is a small, but growing number of cytosolic proteins that are secreted and have no signal peptide. This is referred to as unconventional secretion. Mounting evidence from the past few years suggests that autophagy plays a key role in this type of secretion and this has led to the term secretory autophagy (for a detailed review see Ponpuak et al, 2015). As highlighted above, classical autophagy is a mechanism to degrade cytosolic proteins. The finding that autophagy also contributes to secretion, might be viewed as just another way for the cell to get rid of cytosolic proteins via autophagy. This might be supported by the observation that autophagy-dependent secretion of $\alpha$-synuclein is enhanced when autophagosome-lysosome fusion is inhibited (Ejlerskov et al, 2013). However, this view is not fully correct because most proteins secreted through secretory autophagy are typically not misfolded, aggregated, aged or superfluous. Rather, secretory autophagy is a mechanism by which the cell secrets functional proteins for para- or autocrine signaling. Thus, secretory autophagy is a form of unconventional secretion (Rabouille et al, 2012) that is important for cellular communication. The analogy of secretory autophagy and unconventional secretion is supported by the finding that Golgi matrix proteins of the GRASP family play a role in both unconventional secretion as well as in secretory autophagy in mammals, yeast and Dictyostelium (Rabouille et al, 2012; Kinseth et al, 2007; Duran et al, 2010; Manjihaya et al, 
2010). One of the first and most studied examples of unconventional secretion is IL- $1 \beta$ was also found to be secreted in a manner dependent on ATG5 (Dupont et al, 2011), thus underscoring the notion that secretory autophagy is part of the unconventional secretory pathway.

Little is known so far on the machinery that determines whether an autophagosome will be degradative or secretory. This could be achieved through the acquisition of different vesicles fusion proteins as has been shown in yeast where secretory autophagosome requires the Sso1 SNARE protein to fuse with the plasma membrane while degradative autophagosomes require VAM7/VAM3 to fuse with the vacuole (Duran et al, 2010). Differences seem to also exist at the level of biogenesis, as secretory autophagy was reported to be associated with a specialized membrane microdomain identified in yeast called compartment for unconventional protein secretion (CUPS) identified by the Malhotra group (Bruns et al, 2011). CUPS form under glucose-starvation in close vicinity to ERES, but are devoid of any markers of he ER. CUPS are required for the secretion of Acb1 via the unconventional route. Notably, CUPS biogenesis is not affected by rapamycin, a classical autophagy-inducing stimulus (Bruns et al, 2011). However, later work showed that starvation does induce CUPS formation (Cruz-Garcia et al, 2014), suggesting that CUPS might also represent a specialized form of degradative autophagosomes. In striking difference to classical autophagy nucleation sites, CUPS biogenesis was COPII independent, but instead the membrane source appears to be the Golgi and the TGN or endosomal compartments (CruzGarcia et al, 2014). More work is needed to clarify the definitive role of CUPS in yeast and to understand the fate of autophagosomes generated from CUPS.

Regardless of their true nature in yeast, there is no evidence that CUPS exist in mammalian cells. However, CUPS are structurally very similar to omegasomes because both are induced by starvation and are enriched in PI3P and Atg proteins (Axe et al, 2008; CruzGarcia et al, 2014; Bruns et al, 2011). Therefore, mammalian cells do not have a dedicated source for secretory autophagosomes. This leads us to conclude that specification of autophagosome fate is determined at a later stage. This is supported by the finding that whereas Rab8a is involved in secretory autophagy (Dupont et al, 2011), its close homolog Rab8b appears to be more important for maturation of the degradative autophagosome (Pilli et al, 2012). Further clarification of how secretory autophagy diverges from degradative autophagy came from a study on the secretion of IL-1 $\beta$. In response to lysosomal damage IL$1 \beta$ is recognized by a special autophagy cargo receptor TRIM16, which sorts IL-1 $\beta$ into the 
unconventional secretory pathway (Kimura et al, 2017). TRIM16 interacts with the SNARE protein Sec22b, and recruits IL-1 $\beta$ to LC3-positive membranes. Sec22b then pairs with syntaxin $3 \& 4$ at the plasma membrane to mediate secretion of IL-1 $\beta$, thus showing that secretory autophagy uses a dedicated vesicular fusion machinery that is different from the degradative route that is dependent on syntaxin 17 (Kimura et al, 2017).

What are the roles of secretory autophagy in general biology or in diseases? A role for secretory autophagy in intestinal antimicrobial defense was recently demonstrated. During bacterial infections, Paneth cells secret lysozyme to, which limits bacterial invasion. However, invading bacteria alter the functional organization of the secretory pathway, to inhibit lysozyme secretion. Secretory autophagy was discovered as an alternative pathway that mediates lysozyme secretion under conditions of altered conventional secretion (Bel et al, 2017). Secretory autophagy was triggered by ER stress and required secretion of IL-22 by innate lymphoid cells. Importantly, secretory autophagy was disrupted in Paneth cells expressing Atg16L1 mutants linked Crohn's disease. The potential importance of this finding is that it might explain why mutations found in patients with Crohn's disease often affect the ER stress response and autophagy.

Because the clients of secretory autophagy are functional proteins, it is likely that it is involved in intercellular communication. Cancer associated fibroblasts (CAFs) play an important role in tumor progression, and their secretome has been proposed to promote the growth neighboring cancer cells (Hernandez-Fernaud et al, 2017). Secretory autophagy of CAFs has recently been demonstrated to control the progression of head and neck squamous cell carcinoma (New et al, 2017). Secretory autophagy seems to control the secretion of several cytokines such as IL-6 and IL-8. Therefore, autophagy appears to play non-cell autonomous roles in cancer.

\section{The role of the crosstalk between autophagy and secretion in diseases}

Our understanding of the links between the autophagy and the secretory pathway is still sketchy, and therefore we will limit this section to hypothesize about potential involvement of this crosstalk in diseases. Both the secretory as well as the autophagic pathway are well known to be involved in many diseases such as cancer, neurodegeneration or autoimmune disorders (Galluzzi et al, 2015; Fan et al, 2008; Menzies et al, 2017). Recently, 
a cargo of the secretory autophagy pathway, IL-6, was shown to be involved in tumor cachexia (Pettersen et al, 2017). IL-6 itself was found to induce autophagy in myotubes, which might explain the muscular atrophy in cachectic patients. Serum of cancer patients was found to induce autophagy, which indicates the potential presence of additional factors that are involved in this mechanism. We propose here the following theoretical scenario that requires experimental validation: secreted factors in the serum of cancer patients induce autophagy, thereby inducing secretory autophagy to secret IL-6, which in turn exacerbates systemic autophagy and promotes cachexia. Another example for a role of secretory autophagy in cancer, came from work by the Debnath group, who showed that autophagydependent secretion of IL-6 and the protease MMP2 is an important determinant of the invasive and metastatic potential of Ras-transformed cancer cells (Lock et al, 2014). Knockdown of core autophagy genes was found to decreases invasion, cell motility, and reduces pulmonary metastases, which could be reversed by culturing these knockdown cells in conditioned media of autophagy-competent cells.

Autophagy has been shown to play a crucial role in the defense of epithelial cells against invading pathogens, such as Salmonella or Legionella (Kohler \& Roy, 2017). The autophagy receptor optineurin was previously shown to regulate the ability of cells to clear Salmonella infections (Wild et al, 2011). Optineurin is a further example for a multitasking protein, as it is also known to regulate trafficking of post-Golgi vesicles (Bond et al, 2011). Mutations of optineurin (in particular the E50K mutation) were shown to be associated with normal tension glaucoma and retinopathies (Rezaie et al, 2002; Chi et al, 2010). Mechanistically, this is (at least patially) due to an inability to bind to Rab8 at the trans-Golgi (Chi et al, 2010). Whether autophagy plays a role in these diseases is not known, but we may speculate about this possibility in particular since the small GTPse Rab8 that recruits optineurin to the Golgi, was also shown to be involved in degradative as well as secretory autophagy (Dupont et al, 2011;Pilli et al, 2012). Infection with Salmonella, results in major structural rearrangements of the endomembrane system such as the formation of a Salmonella containing vacuole (SCV) at early stages of infection and large filamentous structures called Salmonella-induced filaments (SIFs) at later stages (Liss \& Hensel, 2015; Personnic et al, 2016). Such structural rearrangements are only possible because the pathogen is using the host trafficking machinery to sculpt membranes and form its replication niche. Rab1 has been introduced above to exert a dual role in autophagy and secretion. Rab1 was shown to be critical for mounting anti-bacterial autophagic defense against Salmonella and this effect was 
reported to be independent of its effects on ER-Golgi trafficking (Huang et al, 2011). Salmonella and Legionella evade autophagy largely by replicating within membrane-bound vacuoles. However, Legionella appears to go one step further as its effector Lpg1137 was recently shown to proteolytically cleave syntaxin 17 and thereby disrupt ER-mitochondria contact sites (Arasaki et al, 2017) that were previously shown to be involved in biogenesis of autophagosomes (Hamasaki et al, 2013). Thus, Legionella is capable of sculpting ER membranes to create its replication niche and at the same time blocks autophagosome formation.

Another spectrum of diseases very frequently linked to the secretory or autophagic pathway is neurodegenerative disorders, in particular Alzheimers disease (AD), Parkinson's disease, or amyotrophic lateral sclerosis (ALS) (Fan et al, 2008; Menzies et al, 2017; Rabouille \& Haase, 2016). One of the major characteristics of AD is the increased accumulation of amyloid $\beta(\mathrm{A} \beta)$ peptide, which is secreted and subsequently aggregates into extracellular $\mathrm{A} \beta$ plaques in $\mathrm{AD}$ brains. However, a role for intracellular accumulation of $\mathrm{A} \beta$ was suggested by the finding that mice lacking ATG7 exhibit defects in the secretion of A $\beta$ (Nilsson et al, 2015; Nilsson et al, 2014; Nilsson et al, 2013). Instead, A $\beta$ accumulated within the cell and formed aggregates in the Golgi. This finding implies that intracellular $A \beta$ accumulation may also contribute to disease progression. Notably, multivesicular bodies (MVB) were negative for $\mathrm{A} \beta$, indicating that ATG7 regulates the transport between the Golgi and MVBs (Nilsson et al, 2015). This tempts to speculate on why is autophagy required for the secretion of $A \beta$ in the first line. This might represent a way for the cell to dispose aggregated (or aggregation prone) protein. This appears counterintuitive, because extracellular deposition of $A \beta$ is a pathogenic factor in $A D$. The precise biologic relevance of this finding remains to be determined. A hallmark of $\mathrm{AD}$ neurons is the fragmentation of the Golgi apparatus, but it is still not fully clear whether this is pathologically relevant. Recent work suggests that this is the case (Joshi et al, 2015). It is also unclear whether this phenotype is linked to the effects on autophagy, and this requires more intense research in the future.

Alpha-synuclein is a neuronal protein that has gained tremendous attention as a major player in the pathogenesis of Parkinson's Disease (Bendor et al, 2013). Mutants of $\alpha$-synuclein form aggregates and appear to disturb membrane-dependent processes. Using a yeast genetics screen to identify suppressors of $\alpha$-synuclein toxicity, the small GTPase Ypt1 (Rab1 in mammals) was identified as a major hit (Cooper et al, 2006). Because few other regulators of ER-to-Golgi trafficking were also found, the authors concluded that anterograde secretory 
trafficking is a process likely to be disrupted by $\alpha$-synuclein. This finding has later been corroborated in mammalian cells (Thayanidhi et al, 2010). The current model is that $\alpha$ synuclein affects the tethering and fusion of COPII vesicles. In addition, $\alpha$-synuclein also potentially disrupts anterograde secretory trafficking within and from the Golgi, because it was found to bind to several Rab GTPases such as Rab8 and Rab3a (Dalfo et al, 2004). In line with the notion that Rab1 is a multitasking protein that regulates both autophagy and secretion, is the observation that $\alpha$-synuclein inhibits autophagy via inhibition of Rab1 activity, and mislocalization of ATG9 (Winslow et al, 2010). Thus, the case of $\alpha$-synuclein represents a good example for how both, autophagy and secretion, might be involved in pathology. Future work is needed to dissect the differential contribution of these two processes to the pathogenesis of Parkinson's disease. Another case that exhibits the autophagy-secretion link in PD comes from work on the Leucine-rich repeat kinase 2 (LRRK2). Recently, LRRK2 was shown to interact with Sec16A and to regulate its targeting to ERES (Cho et al, 2014). Disease causing mutations of LRRK2 perturbed Sec16A localization. LRRK2 appears to be a negative regulator of autophagy, because depletion of LRRK2 increased basal levels of autophagy (Manzoni, 2017). Neurite outgrowth defects linked to mutant LRRK2 expression were dependent on autophagy (Manzoni, 2017). It is unclear how exactly LRRK2 affects autophagy, but it will be interesting to understand how a protein that positively affects secretion, negatively affects autophagy. Understanding the biologic significance of this, will allow us to gain a deeper understanding of the role of LRRK2 in PD.

Amyotrophic lateral sclerosis (ALS) is an incurable neurodegenerative disease with a prominent and well-documented involvement of the autophagic and secretory pathways. Mutations in superoxide dismutase are known to be involved in ALS pathogenesis (Rosen et al, 1993). Motor neurons from mice expressing mutant SOD1 (SOD1 ${ }^{\mathrm{G} 93 \mathrm{~A}}$ ) were show to accumulate large autophagosomes containing ubiquitinated aggregates, indicating a defect in autophagy (Rudnick et al, 2017). Deletion of ATG7, further accelerated neuronal cell death. In support of the role of autophagy in ALS is the fact that several autophagy genes were found to causally underlie ALS (Cirulli et al, 2015). Expression of mutant SOD1 was also shown to inhibit ER-to-Golgi trafficking (Atkin et al, 2014) and to deregulation of trafficking from the Golgi as well as a destabilization of microtubules at the Golgi, leading to fragmentation of this organelle (Bellouze et al, 2016; Haase \& Rabouille, 2015). These alterations of trafficking are thought to be linked to the ER stress found in neurons from ALS patients. Given the prominent role of the autophagic and secretory pathway in ALS, it is surprising that 
only little is known about their crosstalk in disease pathology. Using a Caenorhabditis elegans model, the crosstalk between the cytohesin (an Arf-GEF) was found to interact with SOD1. Inhibition of cytohesin activity, enhanced the autophagic flux and it reduced ER stress, thereby reducing the burden of mutant SOD1 expression (Zhai et al, 2015). The mechanistic details behind this interesting observation need to be identified in future work. An interesting work by the Glimcher group showed that inhibition of ER stress (by XBP1 knockout) restored the autophagic flux, thereby clearing mutant SOD1 aggregates (Hetz et al, 2009). This points towards a crosstalk between ER stress and autophagy that can provide protection against neurodegeneration.

\section{Concluding remarks}

The investigation of the crosstalk between autophagy and the secretory pathway is an emerging field of research. Although significant work has already been done, we still lack mechanistic understanding of how this crosstalk works. For instance, multiple sites on the secretory pathway and multiple trafficking routs were shown to contribute to the biogenesis of autophagosomes. In light of this, it is unclear why the alteration of a single site/route has often such dramatic effects on autophagy. For instance, alterations of ER-PM contact sites strongly affects autophagy (Nascimbeni et al, 2017), which is surprising because we would expect autophagosomes to still form ERES, omegasomes, ER-Mitochondria contact sites or the ERGIC. This raises the possibility that alterations of a single site for autophagosome biogenesis negatively affects the other sites as well. However, it is unclear at the moment whether ER-PM contact sites (or membrane contact sites in general) affect ERES or the ERGIC. Such an interdependent network of membrane may well exist as suggested by recent ultrastructural studies that demonstrate the existence of contact sites between the phagophore and late endosomes, Golgi complex and mitochondria (Biazik et al, 2015). Likewise, it is unclear whether ER export activity affects the formation of contact sites between the ER and other membranes. Future work on autophagosome biogenesis will have to investigate the impact on more than a single nucleation site.

Few RNAi screens have been performed on either the autophagy or the secretory pathway. Despite yielding a large quantity of data, we are still far from a systematic understanding of the crosstalk between these two pathways (Farhan, 2015). This indicates that more research is needed and that we need to combine loss of function screens (RNAi or 
CRISPR) with chemical biology approaches. Recently, a small molecule screen has been conducted to identify stimulators of autophagy that were found to affect some of biologic responses that autophagy regulates (Kuo et al, 2015). Hits from this screen could be used in combination with loss of function conditions to determine the impact on secretion. Moreover, dedicated screening approaches are necessary to investigate the crosstalk of autophagy and secretion.

\section{Acknowledgements}

Work in the laboratory of HF is supported by grants from the Norwegian Cancer Society, the Norwegian Research Council, the Anders Jahre Foundation, and the Swiss Science Foundation. The authors would like to thank Veronika Reiterer for helpful comments on the manuscript.

\section{References}

Appenzeller-Herzog C, Hauri HP. The ER-Golgi intermediate compartment (ERGIC): in search of its identity and function. J Cell Sci. 2006 Jun 1;119(Pt 11):2173-83.

Arasaki K, Mikami Y, Shames SR, Inoue H, Wakana Y, Tagaya M. Legionella effector Lpg1137 shuts down ER-mitochondria communication through cleavage of syntaxin 17. Nat Commun. 2017 May 15;8:15406.

Atkin, J. D., Farg, M. A., Soo, K. Y., Walker, A. K., Halloran, M., Turner, B. J., et al. (2014). Mutant SOD1 inhibits ER-Golgi transport in amyotrophic lateral sclerosis. J. Neurochem. 129, 190-204.

Axe EL, Walker SA, Manifava M, Chandra P, Roderick HL, Habermann A, Griffiths G, Ktistakis NT. Autophagosome formation from membrane compartments enriched in phosphatidylinositol 3-phosphate and dynamically connected to the endoplasmic reticulum. J Cell Biol. 2008 Aug 25;182(4):685-701.

Barlowe C, Schekman R. SEC12 encodes a guanine-nucleotide-exchange factor essential for transport vesicle budding from the ER. Nature. 1993 Sep 23; 365(6444):347-9. 
Behrends C, Sowa ME, Gygi SP, Harper JW. Network organization of the human autophagy system. Nature. 2010 Jul 1; 466(7302):68-76.

Bellouze S, Baillat G, Buttigieg D, de la Grange P, Rabouille C, Haase G. Stathmin 1/2triggered microtubule loss mediates Golgi fragmentation in mutant SOD1 motor neurons. Mol Neurodegener. 2016 Jun 9;11(1):43.

Bel S, Pendse M, Wang Y, Li Y, Ruhn KA, Hassell B, Leal T, Winter SE, Xavier RJ, Hooper LV. Paneth cells secrete lysozyme via secretory autophagy during bacterial infection of the intestine. Science. 2017 Sep 8;357(6355):1047-1052.

Bendor, J. T., Logan, T. P., and Edwards, R. H. (2013). The function of alpha-synuclein. Neuron 79, 1044-1066.

Ben-Tekaya H, Miura K, Pepperkok R, Hauri HP. Live imaging of bidirectional traffic from the ERGIC. J Cell Sci. 2005 Jan 15;118(Pt 2):357-67.

Biazik J, Ylä-Anttila P, Vihinen H, Jokitalo E, Eskelinen EL. Ultrastructural relationship of the phagophore with surrounding organelles. Autophagy. 2015; 11(3):439-51.

Bjørkøy G, Lamark T, Brech A, Outzen H, Perander M, óvervatn A, Stenmark H, Johansen T. p62/SQSTM1 forms protein aggregates degraded by autophagy and has a protective effect on huntingtin-induced cell death. J. Cell Biol. 2005, 171:603-614.

Bond LM, Peden AA, Kendrick-Jones J, Sellers JR, Buss F. Myosin VI and its binding partner optineurin are involved in secretory vesicle fusion at the plasma membrane. Mol Biol Cell. 2011 Jan 1;22(1):54-65.

Bruns C, McCaffery JM, Curwin AJ, Duran JM, Malhotra V. Biogenesis of a novel compartment for autophagosome-mediated unconventional protein secretion. J Cell Biol. 2011 Dec 12; 195(6):979-92.

Cai, H., Yu, S., Menon, S., Cai, Y., Lazarova, D., Fu, C., Reinisch, K., Hay, J. C. and FerroNovick, S. (2007). TRAPPI tethers COPII vesicles by binding the coat subunit Sec23. Nature $445,941-944$.

Carlsson SR, Simonsen A. Membrane dynamics in autophagosome biogenesis. J Cell Sci. 2015 Jan 15;128(2):193-205. 
Chi ZL, Akahori M, Obazawa M, Minami M, Noda T, Nakaya N, Tomarev S, Kawase K, Yamamoto T, Noda S, Sasaoka M, Shimazaki A, Takada Y, Iwata T. Overexpression of optineurin E50K disrupts Rab8 interaction and leads to a progressive retinal degeneration in mice. Hum Mol Genet. 2010 Jul 1;19(13):2606-15.

Cho HJ, Yu J, Xie C, Rudrabhatla P, Chen X, Wu J, Parisiadou L, Liu G, Sun L, Ma B, Ding J, Liu Z, Cai H. Leucine-rich repeat kinase 2 regulates Sec16A at ER exit sites to allow ERGolgi export. EMBO J. 2014 Oct 16;33(20):2314-31.

Cirulli ET, et al., FALS Sequencing Consortium (2015) Exome sequencing in amyotrophic lateral sclerosis identifies risk genes and pathways. Science 347:1436-1441.

Colecchia D, Strambi A, Sanzone S, Iavarone C, Rossi M, Dall'Armi C, Piccioni F, Verrotti di Pianella A, Chiariello M. MAPK15/ERK8 stimulates autophagy by interacting with LC3 and GABARAP proteins. Autophagy. 2012 Dec;8(12):1724-40.

Cooper AA, Gitler AD, Cashikar A, Haynes CM, Hill KJ, Bhullar B, Liu K, Xu K, Strathearn KE, Liu F, Cao S, Caldwell KA, Caldwell GA, Marsischky G, Kolodner RD, Labaer J, Rochet JC, Bonini NM, Lindquist S. Alpha-synuclein blocks ER-Golgi traffic and Rab1 rescues neuron loss in Parkinson's models. Science. 2006 Jul 21;313(5785):324-8.

Cruz-Garcia D, Curwin AJ, Popoff JF, Bruns C, Duran JM, Malhotra V. Remodeling of secretory compartments creates CUPS during nutrient starvation. J Cell Biol. 2014 Dec 22; 207(6):695-703.

Dalfó E, Gómez-Isla T, Rosa JL, Nieto Bodelón M, Cuadrado Tejedor M, Barrachina M, Ambrosio S, Ferrer I. Abnormal alpha-synuclein interactions with Rab proteins in alphasynuclein A30P transgenic mice. J Neuropathol Exp Neurol. 2004 Apr;63(4):302-13.

Davis S, Wang J, Zhu M, Stahmer K, Lakshminarayan R, Ghassemian M, Jiang Y, Miller EA, Ferro-Novick S. Sec24 phosphorylation regulates autophagosome abundance during nutrient deprivation. Elife. 2016 Nov 18;5.

De Duve C, Wattiaux R. Functions of lysosomes. Annu Rev Physiol. 1966;28:435-92.

Dupont N, Jiang S, Pilli M, Ornatowski W, Bhattacharya D, Deretic V. Autophagy-based unconventional secretory pathway for extracellular delivery of IL-1 $\beta$. EMBO J. 2011 Nov 8; 30(23):4701-11. 
Duran JM, Anjard C, Stefan C, Loomis WF, Malhotra V. Unconventional secretion of Acb1 is mediated by autophagosomes. J Cell Biol. 2010 Feb 22; 188(4):527-36.

Ejlerskov P, Rasmussen I, Nielsen TT, Bergström AL, Tohyama Y, Jensen PH, Vilhardt F. Tubulin polymerization-promoting protein (TPPP/p25 $\alpha$ ) promotes unconventional secretion of $\alpha$-synuclein through exophagy by impairing autophagosome-lysosome fusion. J Biol Chem. 2013 Jun 14; 288(24):17313-35.

Fan J, Hu Z, Zeng L, Lu W, Tang X, Zhang J, Li T. Golgi apparatus and neurodegenerative diseases. Int J Dev Neurosci. 2008 Oct;26(6):523-34.

Fan W1, Nassiri A, Zhong Q. Autophagosome targeting and membrane curvature sensing by Barkor/Atg14(L). Proc Natl Acad Sci U S A. 2011 May 10;108(19):7769-74.

Farhan H. Systems biology of the secretory pathway: what have we learned so far? Biol Cell. $2015 \mathrm{Jul} ; 107(7): 205-17$.

Farhan H, Kundu M, Ferro-Novick S. The link between autophagy and secretion: a story of multitasking proteins. Mol Biol Cell. 2017 May 1;28(9):1161-1164.

Farhan H, Reiterer V, Korkhov VM, Schmid JA, Freissmuth M, Sitte HH. Concentrative export from the endoplasmic reticulum of the gamma-aminobutyric acid transporter 1 requires binding to SEC24D. J Biol Chem. 2007 Mar 9;282(10):7679-89.

Farhan H, Weiss M, Tani K, Kaufman RJ, Hauri HP. Adaptation of endoplasmic reticulum exit sites to acute and chronic increases in cargo load. EMBO J. 2008 Aug 6; 27(15):2043-54.

Farré JC, Subramani S. Mechanistic insights into selective autophagy pathways: lessons from yeast. Nat Rev Mol Cell Biol. 2016 Sep;17(9):537-52.

Galluzzi L, Pietrocola F, Bravo-San Pedro JM, Amaravadi RK, Baehrecke EH, Cecconi F, Codogno P, Debnath J, Gewirtz DA, Karantza V, Kimmelman A, Kumar S, Levine B, Maiuri MC, Martin SJ, Penninger J, Piacentini M, Rubinsztein DC, Simon HU, Simonsen A, Thorburn AM, Velasco G, Ryan KM, Kroemer G. Autophagy in malignant transformation and cancer progression. EMBO J. 2015 Apr 1;34(7):856-80.

Gan W, Zhang C, Siu KY, Satoh A, Tanner JA, Yu S. ULK1 phosphorylates Sec23A and mediates autophagy-induced inhibition of ER-to-Golgi traffic. BMC Cell Biol. 2017 May $10 ; 18(1): 22$. 
Ge L, Melville D, Zhang M, Schekman R. The ER-Golgi intermediate compartment is a key membrane source for the LC3 lipidation step of autophagosome biogenesis. Elife. 2013 Aug 6;2:e00947.

Ge L, Zhang M, Kenny SJ, Liu D, Maeda M, Saito K, Mathur A, Xu K, Schekman R. Remodeling of ER-exit sites initiates a membrane supply pathway for autophagosome biogenesis. EMBO Rep. 2017 Sep;18(9):1586-1603.

Graef M, Friedman JR, Graham C, Babu M, Nunnari J. ER exit sites are physical and functional core autophagosome biogenesis components. Mol Biol Cell. 2013 Sep;24(18):2918-31.

Haase G, Rabouille C. Golgi Fragmentation in ALS Motor Neurons. New Mechanisms Targeting Microtubules, Tethers, and Transport Vesicles. Front Neurosci. 2015 Dec 8;9:448.

Hamasaki M, Furuta N, Matsuda A, Nezu A, Yamamoto A, Fujita N, Oomori H, Noda T, Haraguchi T, Hiraoka Y, Amano A, Yoshimori T. Autophagosomes form at ER-mitochondria contact sites. Nature. 2013 Mar 21;495(7441):389-93.

Hansen MD, Johnsen IB, Stiberg KA, Sherstova T, Wakita T, Richard GM, Kandasamy RK, Meurs EF, Anthonsen MW. Hepatitis C virus triggers Golgi fragmentation and autophagy through the immunity-related GTPase M. Proc Natl Acad Sci U S A. 2017 Apr 25;114(17):E3462-E3471.

Hayashi-Nishino M, Fujita N, Noda T, Yamaguchi A, Yoshimori T, Yamamoto A. A subdomain of the endoplasmic reticulum forms a cradle for autophagosome formation. Nat Cell Biol. 2009 Dec;11(12):1433-7.

Hernandez-Fernaud JR, Ruengeler E, Casazza A, Neilson LJ, Pulleine E, Santi A, Ismail S, Lilla S, Dhayade S, MacPherson IR, McNeish I, Ennis D, Ali H, Kugeratski FG, Al Khamici H, van den Biggelaar M, van den Berghe PV, Cloix C, McDonald L, Millan D, Hoyle A, Kuchnio A, Carmeliet P, Valenzuela SM, Blyth K, Yin H, Mazzone M, Norman JC, Zanivan S. Secreted CLIC3 drives cancer progression through its glutathione-dependent oxidoreductase activity. Nat Commun. 2017 Feb 15;8:14206.

He S, Ni D, Ma B, Lee JH, Zhang T, Ghozalli I, Pirooz SD, Zhao Z, Bharatham N, Li B, et al. PtdIns(3)P-bound UVRAG coordinates Golgi-ER retrograde and Atg9 transport by 
differential interactions with the ER tether and the beclin 1 complex. Nat Cell Biol 2013; $15: 1206-1219$.

Hetz C, Thielen P, Matus S, Nassif M, Court F, Kiffin R, Martinez G, Cuervo AM, Brown RH, Glimcher LH. XBP-1 deficiency in the nervous system protects against amyotrophic lateral sclerosis by increasing autophagy. Genes Dev. 2009 Oct 1;23(19):2294-306.

Huang J, Birmingham CL, Shahnazari S, Shiu J, Zheng YT, Smith AC, Campellone KG, Heo WD, Gruenheid S, Meyer T, Welch MD, Ktistakis NT, Kim PK, Klionsky DJ, Brumell JH. Antibacterial autophagy occurs at PI(3)P-enriched domains of the endoplasmic reticulum and requires Rab1 GTPase. Autophagy. 2011 Jan;7(1):17-26.

Ishihara, N., Hamasaki, M., Yokota, S., Suzuki, K., Kamada, Y., Kihara, A., Yoshimori, T., Noda, T. and Ohsumi, Y. Autophagosome requires specific early Sec proteins for its formation and NSF/SNARE for vacuolar fusion. Mol. Biol. Cell 2001, 12: 3690-3702.

Jarvela T, Linstedt AD. Irradiation-induced protein inactivation reveals Golgi enzyme cycling to cell periphery. Journal of Cell Science. 2012;125:973-80.

Joshi G, Bekier ME 2nd, Wang Y. Golgi fragmentation in Alzheimer's disease. Front Neurosci. 2015 Sep 24;9:340.

Joo JH, Wang B, Frankel E, Ge L, Xu L, Iyengar R, Li-Harms X, Wright C, Shaw TI, Lindsten T, Green DR, Peng J, Hendershot LM, Kilic F, Sze JY, Audhya A, Kundu M. The Noncanonical Role of ULK/ATG1 in ER-to-Golgi Trafficking Is Essential for Cellular Homeostasis. Mol Cell. 2016 May 19;62(4):491-506.

Juhasz, G., and T.P. Neufeld. 2006. Autophagy: a forty-year search for a missing membrane source. PLoS Biol. 4:e36.

Kuo, S.Y., Castoreno, A.B., Aldrich, L.N., Lassen, K.G., Goel, G., Dančík, V., Kuballa, P., Latorre, I., Conway, K.L., Sarkar, S., Maetzel, D., Jaenisch, R., Clemons, P.A., Schreiber, S.L., Shamji, A.F., and Xavier, R.J. (2015) Small-molecule enhancers of autophagy modulate cellular disease phenotypes suggested by human genetics. Proc Natl Acad Sci U S A. 112, E4281-E4287.

Liss V, Hensel M. Take the tube: remodelling of the endosomal system by intracellular Salmonella enterica. Cell Microbiol. 2015 May;17(5):639-47. 
Liss V, Swart AL, Kehl A, Hermanns N, Zhang Y, Chikkaballi D, Böhles N, Deiwick J, Hensel M. Salmonella enterica Remodels the Host Cell Endosomal System for Efficient Intravacuolar Nutrition. Cell Host Microbe. 2017 Mar 8;21(3):390-402.

Lock R, Kenific CM, Leidal AM, Salas E, Debnath J. Autophagy-dependent production of secreted factors facilitates oncogenic RAS-driven invasion. Cancer Discov. 2014;4(4):466-79.

Lynch-Day, M. A., Bhandari, D., Menon, S., Huang, J., Cai, H., Bartholomew, C. R., Brumell, J. H., Ferro-Novick, S. and Klionsky, D. J. (2010). Trs85 directs a Ypt1 GEF, TRAPPIII, to the phagophore to promote autophagy. Proc. Natl. Acad. Sci. USA 107, 7811-7816.

Kakuta, S., Yamamoto, H., Negishi, L., Kondo-Kakuta, C., Hayashi, N. and Ohsumi, Y. (2012). Atg9 vesicles recruit vesicle-tethering proteins Trs85 and Ypt1 to the autophagosome formation site. J. Biol. Chem. 287, 44261-44269.

Kakuta S, Yamaguchi J, Suzuki C, Sasaki M, Kazuno S, Uchiyama Y. Small GTPase Rab1B is associated with ATG9A vesicles and regulates autophagosome formation. FASEB J. 2017 Sep;31(9):3757-3773.

Karanasios E, Stapleton E, Manifava M, Kaizuka T, Mizushima N, Walker SA, Ktistakis NT. Dynamic association of the ULK1 complex with omegasomes during autophagy induction. $\mathrm{J}$ Cell Sci. 2013 Nov 15;126(Pt 22):5224-38.

Karanasios E, Walker SA, Okkenhaug H, Manifava M, Hummel E, Zimmermann H, Ahmed Q, Domart MC, Collinson L, Ktistakis NT. Autophagy initiation by ULK complex assembly on ER tubulovesicular regions marked by ATG9 vesicles. Nat Commun. 2016 Aug $11 ; 7: 12420$.

Kimura T, Jia J, Kumar S, Choi SW, Gu Y, Mudd M, Dupont N, Jiang S, Peters R, Farzam F, Jain A, Lidke KA, Adams CM, Johansen T, Deretic V. Dedicated SNAREs and specialized TRIM cargo receptors mediate secretory autophagy. EMBO J. 2017 Jan 4;36(1):42-60.

Kinseth MA, Anjard C, Fuller D, Guizzunti G, Loomis WF, Malhotra V. The Golgiassociated protein GRASP is required for unconventional protein secretion during development. Cell. 2007 Aug 10; 130(3):524-34.

Kohler LJ, Roy CR. Autophagic targeting and avoidance in intracellular bacterial infections. Curr Opin Microbiol. 2017 Feb;35:36-41. 
Krämer A, Mentrup T, Kleizen B, Rivera-Milla E, Reichenbach D, Enzensperger C, Nohl R, Täuscher E, Görls H, Ploubidou A, Englert C, Werz O, Arndt HD, Kaether C. Small molecules intercept Notch signaling and the early secretory pathway. Nat Chem Biol. 2013 Nov;9(11):731-8.

Ktistakis NT. In praise of M. Anselmier who first used the term "autophagie" in 1859. Autophagy. 2017 Aug 24:1-3.

Lemus L, Ribas JL, Sikorska N, Goder V. An ER-Localized SNARE Protein Is Exported in Specific COPII Vesicles for Autophagosome Biogenesis. Cell Rep. 2016 Feb 23;14(7):17101722.

Mancias JD, Goldberg J. Structural basis of cargo membrane protein discrimination by the human COPII coat machinery. EMBO J. 2008 Nov 5;27(21):2918-28.

Manjithaya R, Anjard C, Loomis WF, Subramani S. Unconventional secretion of Pichia pastoris Acb1 is dependent on GRASP protein, peroxisomal functions, and autophagosome formation. J Cell Biol. 2010 Feb 22; 188(4):537-46.

Manzoni C. The LRRK2-macroautophagy axis and its relevance to Parkinson's disease. Biochem Soc Trans. 2017 Feb 8;45(1):155-162.

Matsunaga K, Morita E, Saitoh T, Akira S, Ktistakis NT, Izumi T, Noda T, Yoshimori T. Autophagy requires endoplasmic reticulum targeting of the PI3-kinase complex via Atg14L. J Cell Biol. 2010 Aug 23;190(4):511-21.

Menzies FM, Fleming A, Caricasole A, Bento CF, Andrews SP, Ashkenazi A, Füllgrabe J, Jackson A, Jimenez Sanchez M, Karabiyik C, Licitra F, Lopez Ramirez A, Pavel M, Puri C, Renna M, Ricketts T, Schlotawa L, Vicinanza M, Won H, Zhu Y, Skidmore J, Rubinsztein DC. Autophagy and Neurodegeneration: Pathogenic Mechanisms and Therapeutic Opportunities. Neuron. 2017 Mar 8;93(5):1015-1034.

Miller E, Antonny B, Hamamoto S, Schekman R. Cargo selection into COPII vesicles is driven by the Sec24p subunit. EMBO J. 2002 Nov 15;21(22):6105-13.

Miller EA, Beilharz TH, Malkus PN, Lee MC, Hamamoto S, Orci L, Schekman R. Multiple cargo binding sites on the COPII subunit Sec24p ensure capture of diverse membrane proteins into transport vesicles. Cell. 2003 Aug 22;114(4):497-509. 
Mortimore G.E., Schworer C.M. Induction of autophagy by amino-acid deprivation in perfused rat liver. Nature. 1977;270(5633):174-176.

Nascimbeni AC, Giordano F, Dupont N, Grasso D, Vaccaro MI, Codogno P, Morel E. ERplasma membrane contact sites contribute to autophagosome biogenesis by regulation of local PI3P synthesis. EMBO J. 2017 Jul 14;36(14):2018-2033.

New J, Arnold L, Ananth M, Alvi S, Thornton M, Werner LR, Tawfik O, Dai H, Shnayder Y, Kakarala K, Tsue TT, Girod DA, Ding WX, Anant S, Thomas SM. Secretory autophagy in cancer-associated fibroblasts promotes head and neck cancer progression and offers a novel therapeutic target. Cancer Res. 2017 Sep 28.

Nilsson $\mathrm{P}$, Loganathan $\mathrm{K}$, Sekiguchi $\mathrm{M}$, Matsuba $\mathrm{Y}$. A $\beta$ secretion and plaque formation depend on autophagy. Cell Rep. 2013;5:61-9.

Nilsson P, Sekiguchi M, Akagi T, Izumi S, Komori T, Hui K, Sörgjerd K, Tanaka M, Saito T, Iwata N, Saido TC. Autophagy-related protein 7 deficiency in amyloid $\beta$ (A $\beta$ ) precursor protein transgenic mice decreases $A \beta$ in the multivesicular bodies and induces $A \beta$ accumulation in the Golgi. Am J Pathol. 2015 Feb;185(2):305-13.

Novick P, Field C, Schekman R. Identification of 23 complementation groups required for post-translational events in the yeast secretory pathway. Cell. 1980 Aug; 21(1):205-15.

Novick P, Schekman R. Secretion and cell-surface growth are blocked in a temperaturesensitive mutant of Saccharomyces cerevisiae. Proc Natl Acad Sci U S A. 1979 Apr; 76(4):1858-62.

Orsi A, Razi M, Dooley HC, Robinson D, Weston AE, Collinson LM, Tooze SA. Dynamic and transient interactions of Atg9 with autophagosomes, but not membrane integration, are required for autophagy. Mol Biol Cell. 2012 May;23(10):1860-73.

Oz-Levi D, Ben-Zeev B, Ruzzo EK, Hitomi Y, Gelman A, Pelak K, Anikster Y, Reznik-Wolf H, Bar-Joseph I, Olender T, et al. (2012) Mutation in TECPR2 reveals a role for autophagy in hereditary spastic paraparesis. Am J Hum Genet 91:1065-1072.

Pankiv S, Clausen TH, Lamark T, Brech A, Bruun JA, Outzen H, Overvatn A, Bjorkoy G, Johansen T. p62/SQSTM1 binds directly to Atg8/LC3 to facilitate degradation of ubiquitinated protein aggregates by autophagy. J. Biol. Chem. 2007, 282:24131-24145. 
Papinski D, Schuschnig M, Reiter W, Wilhelm L, Barnes CA, Maiolica A, Hansmann I, Pfaffenwimmer T, Kijanska M, Stoffel I, Lee SS, Brezovich A, Lou JH, Turk BE, Aebersold R, Ammerer G, Peter M, Kraft C. Early steps in autophagy depend on direct phosphorylation of Atg9 by the Atg1 kinase. Mol Cell. 2014 Feb 6;53(3):471-83.

Personnic N, Bärlocher K, Finsel I, Hilbi H. Subversion of Retrograde Trafficking by Translocated Pathogen Effectors. Trends Microbiol. 2016 Jun;24(6):450-62.

Pettersen K, Andersen S, Degen S, Tadini V, Grosjean J, Hatakeyama S, Tesfahun AN, Moestue S, Kim J, Nonstad U, Romundstad PR, Skorpen F, Sørhaug S, Amundsen T, Grønberg BH, Strasser F, Stephens N, Hoem D, Molven A, Kaasa S, Fearon K, Jacobi C, Bjørkøy G. Cancer cachexia associates with a systemic autophagy-inducing activity mimicked by cancer cell-derived IL-6 trans-signaling. Sci Rep. 2017 May 17;7(1):2046.

Pilli M, Arko-Mensah J, Ponpuak M, Roberts E, Master S, Mandell MA, Dupont N, Ornatowski W, Jiang S, Bradfute SB, Bruun JA, Hansen TE, Johansen T, Deretic V. TBK-1 promotes autophagy-mediated antimicrobial defense by controlling autophagosome maturation. Immunity. 2012 Aug 24; 37(2):223-34.

Ponpuak M, Mandell MA, Kimura T, Chauhan S, Cleyrat C, Deretic V. Secretory autophagy. Curr Opin Cell Biol. 2015 Aug;35:106-16.

Puri, C., Renna, M., Bento, C.F., Moreau, K., and Rubinsztein, D.C. (2013) Diverse autophagosome membrane sources coalesce in recycling endosomes. Cell. 154, 1285-1299.

Rabouille C, Haase G. Editorial: Golgi Pathology in Neurodegenerative Diseases. Front Neurosci. 2016 Jan 6;9:489.

Rabouille C, Malhotra V, Nickel W. Diversity in unconventional protein secretion. J Cell Sci. 2012 Nov 15;125(Pt 22):5251-5.

Raote I, Ortega Bellido M, Pirozzi M, Zhang C, Melville D, Parashuraman S, Zimmermann T, Malhotra V. TANGO1 assembles into rings around COPII coats at ER exit sites. J Cell Biol. 2017 Apr 3;216(4):901-909.

Razi M, Chan EY, Tooze SA. Early endosomes and endosomal coatomer are required for autophagy. J Cell Biol. 2009 Apr 20;185(2):305-21. 
Reggiori, F., Wang, C. W., Nair, U., Shintani, T., Abeliovich, H. and Klionsky, D. J. Early stages of the secretory pathway, but not endosomes, are required for Cvt vesicle and autophagosome assembly in Saccharomyces cerevisiae. Mol. Biol. Cell. 2004, 15: 2189-2204.

Rezaie T, Child A, Hitchings R, Brice G, Miller L, Coca-Prados M, Héon E, Krupin T, Ritch R, Kreutzer D, Crick RP, Sarfarazi M. Adult-onset primary open-angle glaucoma caused by mutations in optineurin. Science. 2002 Feb 8; 295(5557):1077-9.

Rosen DR, Siddique T, Patterson D, Figlewicz DA, Sapp P, Hentati A, Donaldson D, Goto J, O'Regan JP, Deng HX, et al. Mutations in $\mathrm{Cu} / \mathrm{Zn}$ superoxide dismutase gene are associated with familial amyotrophic lateral sclerosis. Nature. 1993 Mar 4;362(6415):59-62.

Rowland AA, Voeltz GK. Endoplasmic reticulum-mitochondria contacts: function of the junction. Nature Rev. Mol. Cell Biol. 13, 607-625 (2012).

Rudnick ND, Griffey CJ, Guarnieri P, Gerbino V, Wang X, Piersaint JA, Tapia JC, Rich MM, Maniatis T. Distinct roles for motor neuron autophagy early and late in the SOD $1<$ sup $>$ G93A $</$ sup $>$ mouse model of ALS. Proc Natl Acad Sci U S A. 2017 Sep 26;114(39):E8294-E8303.

Sanchez-Wandelmer J, Ktistakis NT, Reggiori F. ERES: sites for autophagosome biogenesis and maturation? J Cell Sci. 2015 Jan 15;128(2):185-92.

Santos AJ, Raote I, Scarpa M, Brouwers N, Malhotra V. TANGO1 recruits ERGIC membranes to the endoplasmic reticulum for procollagen export. Elife. 2015 Nov 14;4. pii: e10982.

Sasai M, Sakaguchi N, Ma JS, Nakamura S, Kawabata T, Bando H, Lee Y, Saitoh T, Akira S, Iwasaki A, Standley DM, Yoshimori T, Yamamoto M. Essential role for GABARAP autophagy proteins in interferon-inducible GTPase-mediated host defense. Nat Immunol. 2017 Aug;18(8):899-910.

Slobodkin, MR, Elazar Z. The Atg8 family: multifunctional ubiquitin- like key regulators of autophagy. Essays Biochem. 2013, 55, 51-64.

Sprangers J, Rabouille C. SEC16 in COPII coat dynamics at ER exit sites. Biochem Soc Trans 2015, 43:97-103. 
Stadel D, Millarte V, Tillmann KD, Huber J, Tamin-Yecheskel BC, Akutsu M, Demishtein A, Ben-Zeev B, Anikster Y, Perez F, Dötsch V, Elazar Z, Rogov V, Farhan H, Behrends C. TECPR2 Cooperates with LC3C to Regulate COPII-Dependent ER Export. Mol Cell. 2015 Oct 1;60(1):89-104.

Stephens DJ. De novo formation, fusion and fission of mammalian COPII-coated endoplasmic reticulum exit sites. EMBO Rep. 2003 Feb;4(2):210-7.

Svenning S, Johansen T. Selective autophagy. Essays Biochem. 2013;55:79-92.

Takeshige, K., Baba, M., Tsuboi, S., Noda, T., and Ohsumi, Y. (1992) Autophagy in yeast demonstrated with proteinase-deficient mutants and conditions for its induction. J. Cell. Biol. $119,301-311$.

Thayanidhi, N., Helm, J. R., Nycz, D. C., Bentley, M., Liang, Y., and Hay, J. C. (2010). Alpha-synuclein delays endoplasmic reticulum (ER)-to-Golgi transport in mammalian cells by antagonizing ER/Golgi SNAREs. Mol. Biol. Cell. 21, 1850-1863.

Wang, J., Menon, S., Yamasaki, A., Chou, H. T., Walz, T., Jiang, Y. and Ferro-Novick, S. (2013). Ypt1 recruits the Atg1 kinase to the preautophagosomal structure. Proc. Natl. Acad. Sci. USA 110, 9800-9805.

Weidberg, H., Shvets, E., Shpilka, T., Shimron, F., Shinder, V., and Elazar, Z. (2010) LC3 and GATE-16/GABARAP subfamilies are both essential yet act differently in autophagosome biogenesis. EMBO J. 29, 1792-1802.

Wild P, Farhan H, McEwan DG, Wagner S, Rogov VV, Brady NR, Richter B, Korac J, Waidmann O, Choudhary C, Dötsch V, Bumann D, Dikic I. Phosphorylation of the autophagy receptor optineurin restricts Salmonella growth. Science. 2011 Jul 8;333(6039):228-33.

Winslow AR, Chen CW, Corrochano S, Acevedo-Arozena A, Gordon DE, Peden AA, Lichtenberg M, Menzies FM, Ravikumar B, Imarisio S, Brown S, O'Kane CJ, Rubinsztein DC. $\alpha$-Synuclein impairs macroautophagy: implications for Parkinson's disease. J Cell Biol. 2010 Sep 20;190(6):1023-37.

Yamamoto, H., Kakuta, S., Watanabe, T.M., Kitamura, A., Sekito, T., Kondo- Kakuta, C., Ichikawa, R., Kinjo, M., and Ohsumi, Y. (2012). Atg9 vesicles are an important membrane source during early steps of autophagosome formation. J. Cell Biol. 198, 219-233. 
Yamasaki, A., Menon, S., Yu, S., Barrowman, J., Meerloo, T., Oorschot, V., Klumperman, J., Satoh, A. and Ferro-Novick, S. (2009). mTrs130 is a component of a mammalian TRAPPII complex, a Rab1 GEF that binds to COPI-coated vesicles. Mol. Biol. Cell 20, 4205-4215.

Ylä-Anttila P, Vihinen H, Jokitalo E, Eskelinen EL. 3D tomography reveals connections between the phagophore and endoplasmic reticulum. Autophagy. 2009 Nov;5(8):1180-5.

Young AR, Chan EY, Hu XW, Köchl R, Crawshaw SG, High S, Hailey DW, LippincottSchwartz J, Tooze SA. Starvation and ULK1-dependent cycling of mammalian Atg9 between the TGN and endosomes. J Cell Sci. 2006 Sep 15;119(Pt 18):3888-900.

Zacharogianni M, Kondylis V, Tang Y, Farhan H, Xanthakis D, Fuchs F, Boutros M, Rabouille C. ERK7 is a negative regulator of protein secretion in response to amino-acid starvation by modulating Sec16 membrane association. EMBO J. 2011 Aug 16;30(18):3684700.

Zaffagnini G, Martens S. Mechanisms of Selective Autophagy. J Mol Biol. 2016 May 8; 428(9Part A): 1714-1724.

Zeuschner D, Geerts WJ, van Donselaar E, Humbel BM, Slot JW, Koster AJ, Klumperman J. Immuno-electron tomography of ER exit sites reveals the existence of free COPII-coated transport carriers. Nat Cell Biol. 2006 Apr;8(4):377-83.

Zhai J, Zhang L, Mojsilovic-Petrovic J, Jian X, Thomas J, Homma K, Schmitz A, Famulok M, Ichijo H, Argon Y, Randazzo PA, Kalb RG. Inhibition of Cytohesins Protects against Genetic Models of Motor Neuron Disease. J Neurosci. 2015 Jun 17;35(24):9088-105.

Zhao S, Li CM, Luo XM, Siu GK, Gan WJ, Zhang L, Wu WK, Chan HC, Yu S. Mammalian TRAPPIII Complex positively modulates the recruitment of Sec13/31 onto COPII vesicles. Sci Rep. 2017 Feb 27;7:43207.

Zheng YT, Shahnazari S, Brech A, Lamark T, Johansen T, Brumell JH. The adaptor protein p62/SQSTM1 targets invading bacteria to the autophagy pathway. J Immunol. 2009 Nov 1;183(9):5909-16.

Zhou C, Ma K, Gao R, Mu C, Chen L, Liu Q, Luo Q, Feng D, Zhu Y, Chen Q. Regulation of mATG9 trafficking by Src- and ULK1-mediated phosphorylation in basal and starvationinduced autophagy. Cell Res. 2017 Feb;27(2):184-201. 
Zoppino FC, Militello RD, Slavin I, Alvarez C, Colombo MI. Autophagosome formation depends on the small GTPase Rab1 and functional ER exit sites. Traffic 2010, 11:1246-1261.

\section{Figure legends}

Figure 1. The secretory and autophagic pathway. $\boldsymbol{A}$, Schematic representation of the organelles and vesicles in the ER-Golgi system together with a representation of the COPII budding process. $\boldsymbol{B}$, The autophagy cascade. $\boldsymbol{C}$, A selection of some types of selective autophagy.

Figure 2. Models for the role of the secretory pathway in autophagy. $\boldsymbol{A}$, The secretory pathway (in this case represented by ERES) act as an assembly platform from where autophagosomes are formed. $\boldsymbol{B}$, ERES donate COPII vesicles that transfer membranes and proteins to the autophagy initiation site and thereby facilitate formation of autophagosomes. $\boldsymbol{C}$, The secretory pathway (e.g. ERES or ERGIC) bud vesicles that fuse with autophagosomes and thereby contribute to their maturation. D, ERES contribute towards the autophagy initiation site, which in turn recruits ATG9 vesicles arriving from the Golgi and post-Golgi compartments (not depicted here). 